\title{
Fibroepithelioma of Pinkus: Solitary tumor or sign of a complex gastrointestinal syndrome
}

\author{
CATERINA LONGO ${ }^{1}$, GIOVANNI PELLACANI ${ }^{2}$, ALDO TOMASI $^{3}$, \\ VICTOR DESMOND MANDEL ${ }^{2}$ and GIOVANNI PONTI ${ }^{3}$ \\ ${ }^{1}$ Dermatology and Skin Cancer Unit, Arcispedale Santa Maria Nuova-IRCCS, I-42123 Reggio Emilia; \\ Departments of ${ }^{2}$ Dermatology and ${ }^{3}$ Clinical and Diagnostic Medicine and Public Health, \\ University of Modena and Reggio Emilia, I-41100 Modena, Italy
}

Received July 23, 2015; Accepted October 23, 2015

DOI: $10.3892 / \mathrm{mco} .2016 .794$

\begin{abstract}
Fibroepithelioma of Pinkus (FEP), which is considered to be an uncommon variant of basal cell carcinoma, has been described in association with other systemic diseases. However, no specific studies are currently available on this subject. The aim of our study was to evaluate the clinical and morphological characteristics of FEP and investigate whether this rare tumor is a single entity or seen in the context of a more complex syndrome. We retrospectively analyzed 49 cases of FEP diagnosed and excised in a single academic institution from 1995 to 2011 . The tumors were mainly located on the trunk $(77.55 \%)$, followed by the lower extremities $(12.20 \%)$ and the head and neck (10.20\%). In 9 of the 49 cases (18\%), FEP was associated with gastrointestinal tumors. The abovementioned cases are presented in an attempt to make clinicians more aware of a possible association between FEP and gastrointestinal cancer. Although a possible underlying common genetic background between FEP and gastrointestinal tumors was not provided, our study suggests that patients with FEP should be screened for the occurrence of gastrointestinal tumors.
\end{abstract}

\section{Introduction}

Fibroepithelioma of Pinkus (FEP) is considered to be an uncommon variant of basal cell carcinoma (BCC) (1), although it was recently described as a trichoblastoma $(2,3)$. Clinically, FEP appears as a solitary non-pigmented or brownish to gray, well-outlined flat or polypoid lesion, with peculiar

Correspondence to: Dr Giovanni Ponti, Department of Clinical and Diagnostic Medicine and Public Health, University of Modena and Reggio Emilia, 71 Via del Pozzo, I-41100 Modena, Italy

E-mail: giovanni.ponti@unimore.it

Key words: Cowden syndrome, fibroepithelioma of Pinkus, gastrointestinal tumors, Gorlin-Goltz syndrome, Patched 1 gene mutations, syndromic setting dermoscopic and confocal aspects (4) and with a predilection for the lower trunk (Fig. 1).

Histopathologically, FEP is characterized by numerous thin anastomosing cords of basaloid cells extending downward from the epidermis into the dermis within a fibrotic stroma (Fig. 1).

Unlike the common types of BCC, FEP exhibits an overwhelming predilection for the trunk (particularly the lower back) and the extremities, with only $5 \%$ of the tumors arising on sun-damaged skin. The clinical differential diagnosis includes a wide spectrum of lesions, such as skin tags or dermal nevi. However, the diagnosis is easier when the clinical examination is combined with dermoscopy and confocal microscopy $(4,5)$.

Although FEP was originally described by Pinkus as a variant of $\mathrm{BCC}$, the nature of this tumor remains a subject of debate.

The strong predilection for the lumbar region (not sun-exposed area) and for the female gender differentiates FEP from other BCC subtypes that usually arise on sun-exposed areas and/or predominantly in men.

Furthermore, FEP exhibits a peculiar histopathological architecture, as well as the presence of Merkel cells, which are usually absent in BCCs but found in trichoblastomas.

Thus far, FEP has been described as a solitary lesion or in association with Paget's disease in a limited number of case series. However, no studies have been performed to investigate whether this lesion arises as an isolated rare tumor or in patients harboring specific diseases.

The aim of our study was the characterization of a consecutive series of FEPs to identify a possible association of this tumor with concomitant diseases.

\section{Patients and methods}

Cases. All the histopathologically-proven cases of FEP excised in a single referral center (Department of Dermatology, University of Modena and Reggio Emilia, Modena, Italy) from 1995 to 2011 were retrospectively reviewed. Clinical data including gender, location and age were recorded. Location was divided into head and neck, trunk, back, genitalia, upper extremities and lower extremities. 

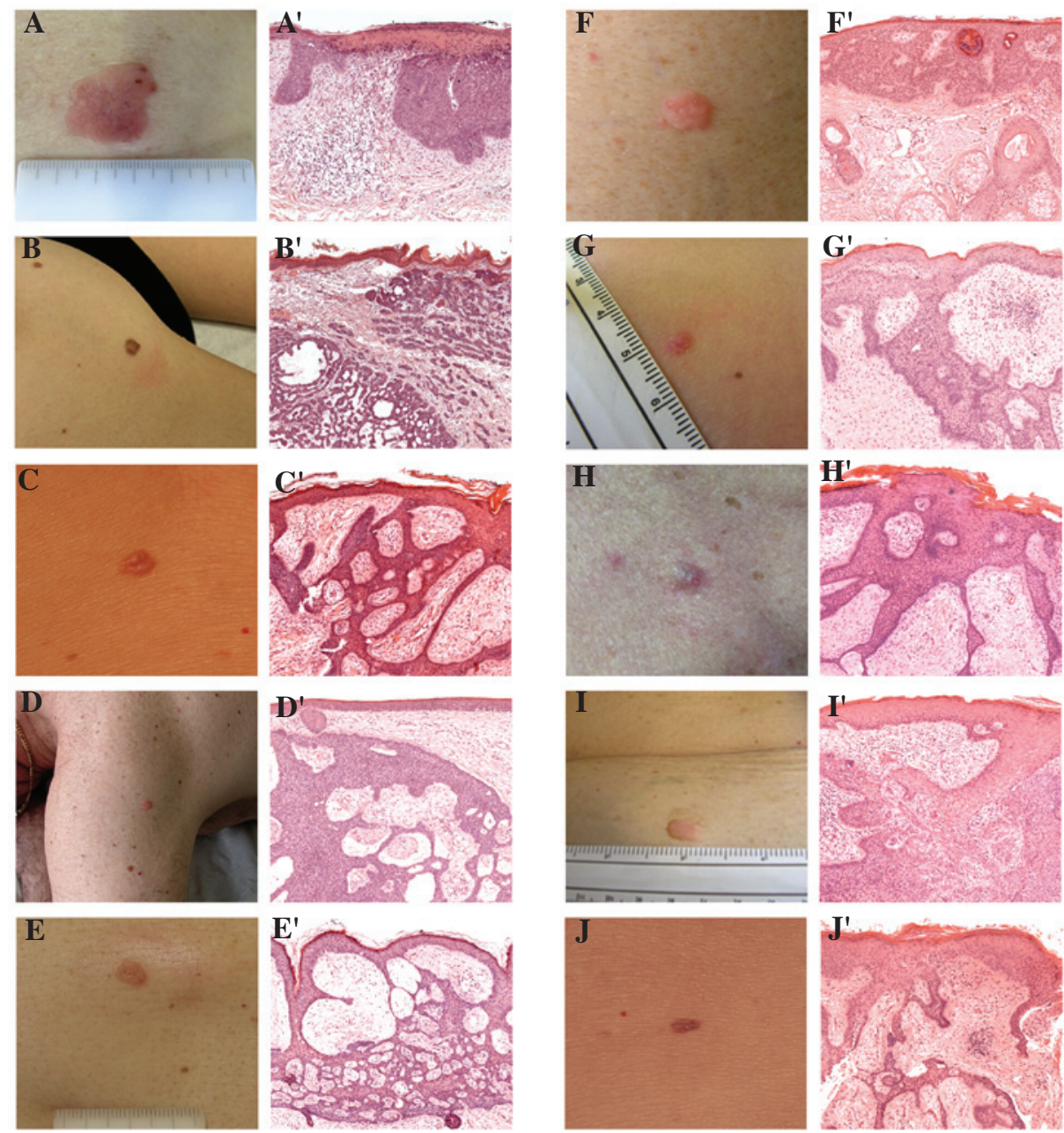

Figure 1. (A-J) Clinical and histological images of fibroepitheliomas of Pinkus (FEPs) collected in our Department. Clinically, FEP appears as a solitary non-pigmented, flat or polypoid lesion, most commonly localized on the trunk or the extremities: (A) Male, aged 50 years, with FEP of the trunk; (B) female, aged 36 years, with FEP of the trunk; (C) female, aged 32 years, with FEP of the left lower limb; (D) male, aged 63 years, with FEP of the left shoulder; (E) female, aged 38 years, with a FEP of the right lower limb; (F) male, aged 47 years, with a FEP of the trunk; (G) female, aged 39 years, with FEP of the left lower limb; (H) male, aged 59 years, with FEP of the trunk; (I) female, aged 44 years, with FEP of the trunk; and (J) female, aged 34 years, with FEP of the trunk. (A'-J') Histopathological images of the respective lesions: FEP exhibits numerous long, thin, branching and anastomosing cords of basaloid cells within a fibrotic stroma. Hematoxylin and eosin staining; original magnification, x5.

The data on excised tumors (skin tumors or visceral malignancies) were retrieved from the database of the Department of Pathology at the University of Modena and Reggio Emilia.

Moreover, the medical records of all the patients, such as radiological examinations or surgical procedures, were reviewed to obtain a complete picture of each patient's health status. The present study was conducted in accordance with the principles of the Declaration of Helsinki.

This study's protocol and the submitted manuscript have been approved by the Institutional Review Board of the Department of Surgery, School of Medicine, Dentistry and Morphological Sciences, University of Modena and Reggio Emilia (Modena, Italy).

\section{Results}

Association of FEP with cancer. A total of 49 cases of FEP were identified, mainly located on the trunk (77.55\%), followed by the lower extremities (12.20\%) and the head and neck $(10.20 \%)$. The mean age of the patients was 64.04 years and 31 of the patients were female (63\%). The tumors found in patients with FEP are listed in Table I. Of the 49 patients, $9(18 \%)$ were found to have gastrointestinal tumors, 4 patients (8\%) had breast carcinoma and 7 patients $(14 \%)$ had other cancers. More specifically, 6 patients had colonic adenocarcinoma and 3 patients had neuroendocrine gastrointestinal tumors. Of the 49 patients, 1 (3\%) was affected with mammary Paget's disease and 1 with perianal extramammary Paget's dissease.

In all but 2 cases, FEP appeared 1-2 years prior to the internal malignancies, whereas in these 2 cases the malignancies occurred synchronously with FEP.

\section{Discussion}

FEP is a rare tumor that is considered to be a variant of BCC, although its classification remains to be clarified. Moreover, the specific skin location and gender predilection differentiates this tumor from 'classical' BCC. Furthermore, BCC and FEP share certain characteristics, such as the expression of 
Table I. Non-skin tumors diagnosed in patients with fibroepithelioma of Pinkus.

\begin{tabular}{|c|c|c|c|}
\hline \multirow[b]{2}{*}{ Tumors } & \multicolumn{2}{|c|}{ Gender } & \multirow[b]{2}{*}{$\begin{array}{c}\text { Total } \\
\mathrm{n}=49(100 \%)\end{array}$} \\
\hline & $\begin{array}{c}\text { Male } \\
\mathrm{n}=18(37 \%)\end{array}$ & $\begin{array}{c}\text { Female, } \\
n=31(63 \%)\end{array}$ & \\
\hline Gastrointestinal tumors & $5(28)$ & $4(13)$ & $9(18)$ \\
\hline Upper & $1(20)$ & $1(25)$ & $2(22)$ \\
\hline Lower & $4(80)$ & $3(75)$ & $7(78)$ \\
\hline Colonic Adenocarcinoma & $3(60)$ & $3(75)$ & $6(67)$ \\
\hline Neuroendocrine gastrointestinal tumor & $2(40)$ & $1(25)$ & $3(33)$ \\
\hline Extramammary perianal Paget's disease & $0(0)$ & $1(3)$ & $1(2)$ \\
\hline Mammary Paget's disease & $0(0)$ & $1(3)$ & $1(2)$ \\
\hline Breast carcinoma & $0(0)$ & $4(13)$ & $4(8)$ \\
\hline Other cancers & $4(22)$ & $3(10)$ & $7(14)$ \\
\hline
\end{tabular}

androgen receptor, which is minimal in trichoblastomas (6). However, FEPs and trichoblastomas display Merkel cells, which are usually absent in BCC (7).

In the present study, we observed an association between FEP and gastrointestinal tumors. FEP was previously reported in the literature in association with Paget's disease (7), breast cancer $(8,9)$ and, as a variant of BCC, Gorlin-Goltz syndrome (10). However, conclusive epidemiological and biomolecular data on these clinical associations are currently lacking $(11,12)$.

Considering our data, we may hypothesize a pathogenetic association between FEP and gastrointestinal neuroendocrine tumors, due to the common embryogenic origin of Merkel cells and neuroendocrine cells from the neural crest. Similar to the well-known genodermatosis (13), a common aberration of genes implicated in mesoectodermal differentiation may play a role in the pathogenesis of FEPs and gastrointestinal adenocarcinomas. Mutation in the tumor suppressor gene TP53 may predispose to the development of FEP (14). Similar to BCC, it has been hypothesized that mutations in the Patched 1 gene (PTCH1), which is responsible for inhibitory signaling in the Hedgehog pathway, may also lead to the development of FEP.

In our study, we did not investigate for PTCH1 mutations or another possible genetic background, although the association of FEP with gastrointestinal tumors appears to be part of a more complex, although still not well defined phenotype, rather than a coincidental finding.

Gastrointestinal tumors may be associated with either Gorlin-Goltz syndrome or trichilemmal lesions (13). As regards the latter condition, Cowden syndrome is characterized by the occurrence of several tumors, including breast, endometrial, thyroid, kidney and colorectal cancers, in association with dermatological manifestations such as oral/skin papillomas and trichilemmomas, and with PTEN mutations (13). Interestingly, dermatological signs may occur prior to systemic symptoms (13). If we consider FEP as a trichoblastoma, it appears more likely that this rare skin tumor should be considered to be part of the cutaneous spectrum of Cowden syndrome (in which hamartomas and colon carcinomas have been recently described) (15), or as a tumor associated with a new syndrome including gastrointestinal tumors. Furthermore, our cases were characterized by early onset of FEP followed by the development of gastrointestinal tumors. This peculiar onset of dermatological diseases may be seen in the context of genodermatosis (12).

Although a possible underlying common genetic substrate between FEP and gastrointestinal tumors was not identified, our study included a consecutive large series of FEPs from a single academic institution, with a careful examination of the patients' medical history. However, additional cases are required to confirm this association and assess its predictive value. It is recommended that FEP patients are clinically evaluated and screened for gastrointestinal tumors. Our clinico-epidemiological findings raise the question of whether FEP is a single entity or part of a more complex and not yet defined gastrointestinal syndrome.

\section{Acknowledgements}

All the authors made significant intellectual contributions to this study and each author listed has read and approved the submitted manuscript.

\section{References}

1. Pinkus H: Premalignant fibroepithelial tumors of skin. AMA Arch Dermatol Syphilol 67: 598-615, 1953.

2. Bowen AR and LeBoit PE: Fibroepithelioma of Pinkus is a fenestrated trichoblastoma. Am J Dermatopathol 27: 149-154, 2005.

3. Sellheyer K, Nelson P and Kutzner H: Fibroepithelioma of Pinkus is a true basal cell carcinoma developing in association with a newly identified tumour-specific type of epidermal hyperplasia. Br J Dermatol 166: 88-97, 2012.

4. Longo C, Soyer HP, Pepe P, Casari A, Wurm EM, Guitera P and Pellacani G: In vivo confocal microscopic pattern of fibroepithelioma of Pinkus. Arch Dermatol 148: 556, 2012.

5. Zalaudek I, Ferrara G, Broganelli P, Moscarella E, Mordente I, Giacomel J and Argenziano G: Dermoscopy patterns of fibroepithelioma of Pinkus. Arch Dermatol 142: 1318-1322, 2006.

6. Katona TM, Ravis SM, Perkins SM, Moores WB and Billings SD: Expression of androgen receptor by fibroepithelioma of Pinkus: Evidence supporting classification as a basal cell carcinoma variant? Am J Dermatopathol 29: 7-12, 2007.

7. Warner TF, Burgess $\mathrm{H}$ and Mohs FE: Extramammary Paget's disease in fibroepithelioma of Pinkus. J Cutan Pathol 9: 340-344, 1982. 
8. Bryant J: Fibroepithelioma of Pinkus overlying breast cancer. Arch Dermatol 121: 310, 1985.

9. Sandbank M and Baschan D: Multiple trichoepitelioma and breast carcinoma. Simultaneous appearance of epithelioma adenoides cysticum and infiltrating lobular carcinoma of the breast. Arch Dermatol 114: 1230, 1978

10. Gorlin RJ and Goltz RW: Multiple nevoid basal-cell epithelioma, jaw cysts and bifid rib. A syndrome. N Engl J Med 262: 908-912, 1960.

11. Prodinger PM, Sarbia M, Massmann J, Straka C, Meyer G and Steinlein OK: Gorlin syndrome associated with small bowel carcinoma and mesenchymal proliferation of the gastrointestinal tract: Case report and review of literature. BMC Cancer 10: 360, 2010 .
12. Ponti G, Pellacani G, Seidenari S, Pollio A, Muscatello U and Tomasi A: Cancer-associated genodermatoses: Skin neoplasms as clues to hereditary tumor syndromes. Crit Rev Oncol Hematol 85: 239-256, 2013.

13. Saldanha G, Fletcher A and Slater DN: Basal cell carcinoma: A dermatopathological and molecular biological update. Br J Dermatol 148: 195-202, 2003.

14. Sidhu GS, Chandra P and Cassai ND: Merkel cells, normal and neoplastic: An update. Ultrastruct Pathol 29: 287-294, 2005.

15. Stanich PP, Owens VL, Sweetser S, Khambatta S, Smyrk TC, Richardson RL, Goetz MP and Patnaik MM: Colonic polyposis and neoplasia in Cowden syndrome. Mayo Clin Proc 86: 489-492, 2011. 